\title{
Inflammatory Myofibroblastic Bladder Tumor in a Patient with Von Recklinghausen's Syndrome
}

\section{Eleftherios Chatzidarellis, ${ }^{1}$ Evangelos Mazaris, ${ }^{1}$ Andreas Skolarikos, ${ }^{1}$ Demonakou Maria, ${ }^{2}$ Iraklis Mitsogiannis, ${ }^{1}$ Nafsika Mousiou, ${ }^{2}$ and Apostolos Bisas ${ }^{1}$}

${ }^{1}$ 2nd Department of Urology Athens Medical School, Sismanoglio Hospital, Sismanogliou 1, 15126 Marousi, Athens, Greece

${ }^{2}$ Department of Pathology, Sismanoglio Hospital, Sismanogliou 1, 15126 Marousi, Athens, Greece

Correspondence should be addressed to Eleftherios Chatzidarellis, ehatzid@yahoo.gr

Received 25 April 2010; Accepted 16 July 2010

Academic Editor: Yigal Dror

Copyright (C) 2010 Eleftherios Chatzidarellis et al. This is an open access article distributed under the Creative Commons Attribution License, which permits unrestricted use, distribution, and reproduction in any medium, provided the original work is properly cited.

\begin{abstract}
Myofibroblastic tumor, also known as inflammatory pseudotumor or pseudosarcoma, is a benign tumor with mesenchymal origin. Bladder location is very uncommon. We report the case of a 58-year-old man with a history of von Recklinghausen's disease who complained for painless macroscopic hematuria 5 months after suprapubic prostatectomy. The radiograph evaluation revealed a bladder tumor, and the pathologic examination following a transurethral resection showed inflammatory myofibroblastic tumor of the bladder. The patient finally underwent a radical cystectomy due to the uncertain pathogenesis of inflammatory myofibroblastic tumor as well as the rarity of cases published on bladder tumors in Von Recklinghausen's patients.
\end{abstract}

\section{Introduction}

Inflammatory myofibroblastic tumor (IMT) or inflammatory pseudotumor (IP) of the urinary bladder is a rare benign nonepithelial lesion. This type of tumor has been described in several organs and anatomical sites including lung, head, neck, and brain. In the genitourinary system, the tumor usually originates in the bladder [1], but it has also been reported in the kidney, urethra, prostate, ureter, and rete testis. It was Roth in 1980 who first described such a lesion in the genitourinary tract [2], followed by Proppe et al. who reported similar findings in eight patients who had recently undergone a surgical procedure. The term "postoperative spindle cell nodule" (PSCN) was used to define the reactive process within three months of a surgical procedure at the site it derives [3]. Inflammatory pseudotumor (IP) on the other hand is histologically similar to PSCN, but the diagnosis is made in the absence of prior surgery [4, 5]. Regarding patient's prognosisa it is important to distinguish and differentiate this lesion from sarcoma, fact that can be difficult both clinically and histologically. The case presented here is the first of IMT in a patient with Von Recklinghausen's neurofibromatosis.

\section{Case Presentation}

A fifty-eight-year old man was evaluated for painless macroscopic hematuria. The patient had a family history of von Recklinghausen's disease with the typical multiple cutaneous neurofibromas. Initial abdominal ultrasound revealed a tumor on the bladder dome which was confirmed by a subsequent cystoscopy. A CT scan of the abdomen showed no perivesical tissue extension, no lymph node enlargement and no hydronephrosis presence (Figure 1). Due to its uncommon endoscopically features and extension of the lesion (large base, smooth surface, and approximate size $>3 \mathrm{~cm}$ ) the patient underwent an incomplete transurethral resection of the tumor for staging purposes and to define its origin. Histopathology following tumor resection depicted a neoplasm of mesenchymal origin with immunohistochemical findings indicating the diagnosis of inflammatory myofibroblastic tumor (IMT). Vimentin was diffusely expressed in neoplastic cells and desmin, $\alpha$-SMA, s-100, CD117, CD34 and keratins of low molecular weight were focally positive. (Figures 2(a) and 2(b)). On the other hand CD30, NSE and keratins of high molecular weight were not expressed at all. 


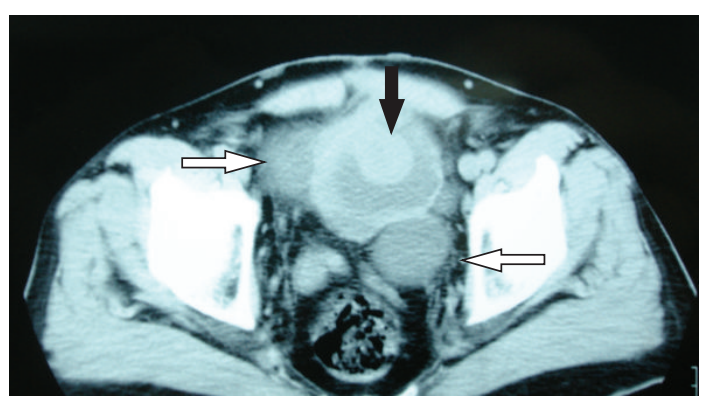

Figure 1: Computed tomography of the lower abdomen showing an exophytic lesion arising from the dome of the bladder (black arrow) and two large diverticulums (white arrows).

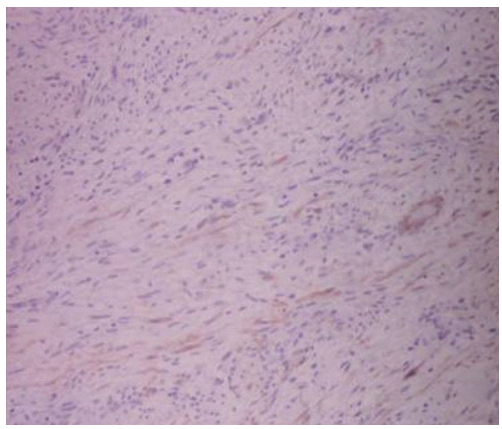

(a)

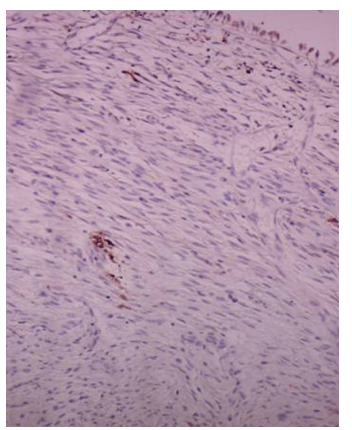

(b)

FIgURE 2: The immunohistochemical staining profile of the tumor (a) APAP/ a-SMA X 25, and (b) APAP/S-100 X 25.

Interestingly, our patient had a recent 5-month history of suprapubic prostatectomy due to lower urinary tract symptoms, during which no bladder wall pathology was recorded. In addition, preprostatectomy imaging was reviewed and quoted as normal. Although the diagnosis of inflammatory pseudotumor could be made, the patient was consented for the uncertain biological behavior and the malignant potential of the tumor. Upon discussing definitive treatment the patient decided to undergo radical cystectomy. As a consequence, radical cystectomy followed by an ileal conduit was performed. Additionally due to lack of genetic test to confirm his inherited disease, the excised cutaneous lesions from the site of the performed stoma were sent for histopathologic evaluation (Figure 3). Macroscopic view of the cystectomy specimen revealed two polypoid lesions with smooth, elastic surface, 1 and $1,5 \mathrm{~cm}$ in diameter located at the bladder dome (Figure 4(a)). The ulcerated surface of the polypoid lesion was recognized microscopically (Figure 4(b)). The microscopic and immunochemistry findings confirmed the initial diagnosis after the transurethral resection of the lesion.

\section{Discussion}

Inflammatory myofibroblastic tumor or pseudotumor is a rare neoplasm believed to be benign. Among the genitourinary organs, the bladder is more commonly affected; still, this entity is extremely rare. Adult males are predominated but the disease can also affect children and elderly patients. Painless hematuria is the most common presenting symptom followed by lower urinary tract symptoms and chronic pelvic pain. Rarely does it constitute an incidental finding during radiological examination. Predisposing or exacerbating factors of genitourinary manifestation include cigarette smoking and prior surgery in the form of an endoscopic bladder surgery or an open gynecological intervention of the pelvis $[6,7]$.

IMT should be differentiated from benign lesions such as leiomyoma or solitary fibrous tumors (SFTs), and malignant lesions such as leiomyosarcoma, sarcomatoid carcinoma, or rhabdomyosarcoma. These entities share common histopathological findings. However, their clinical behavior is entirely different. To properly differentiate immunochemistry is essential. Anaplastic lymphoma kinase (ALK) which stains positive in approximately half of the IMT cases is a promising marker under investigation [1].

Neurofibromatosis type-1 (NF-1) is a familial autosomal dominant syndrome characterized by multiple cutaneous neurofibromas and café au lait spots, skeletal abnormalities, and nervous system manifestations. Urinary tract is rarely involved with the most common manifestation being the development of neurofibromas [8]. Two cases of nonepithelial bladder tumors, one leiomyosarcoma [9] and one solitary leiomyoma [10], have been published. Alterations in the expression of NF-1 gene and of several growth factors may explain the development of mesenchymal tumors in these patients. Our patient has also been operated on and this could activate the process of tumor formation, according to the term "postoperative spindle cell nodule."

Bladder inflammatory myofibroblastic tumors follow a benign indolent course are treated conservatively by transurethral resection or partial cystectomy. Followup constitutes of repeated cystoscopies and biopsies to confirm complete disease remission. When IMT is clearly distinguished from aggressive lesions such as sarcomas radical surgery, radiation or chemotherapy could be considered as overtreatment $[1,7]$.

The patient was fully informed and consented to undergo radical cystectomy. The decision was based on the rarity and the unknown natural history of IMT as well as the scarce cases published on bladder tumors in Von Recklinghausen's patients. Alternative therapeutic approaches could be transurethral resection of the tumor or partial cystectomy. However the relapse rate following these treatments ranges 


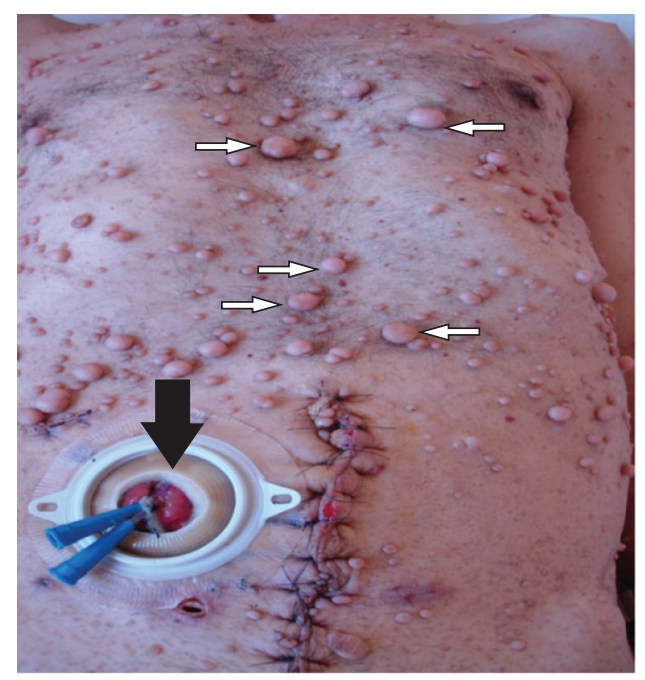

(a)

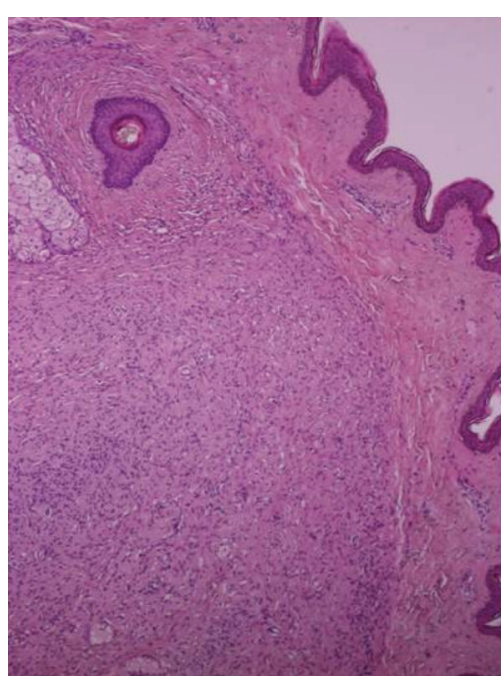

(b)

Figure 3: (a) Numerous cutaneous neurofibromas (small white arrows) and the performance of the ileal stoma (black arrow) located below the right line between umbilicus and the anterior superior iliac spine. (b) Microscopic view of resected cutaneous neurofibroma at the site of the performed stoma (H-E stain X 2.5).

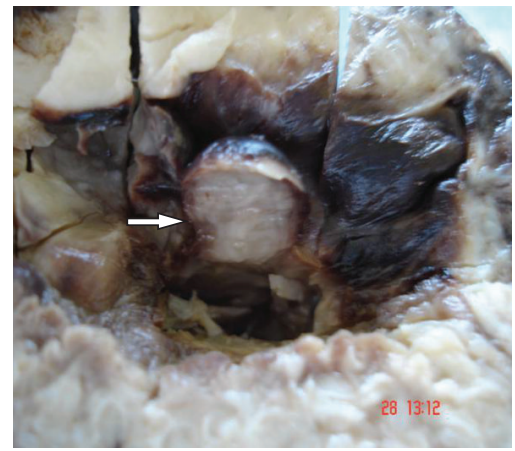

(a)

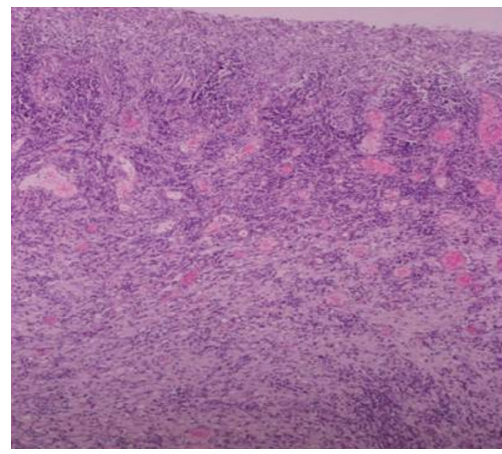

(b)

FIGURE 4: (a) Intraluminal macroscopic view of cystectomy specimen. Recognition of one out of the two polypoid lesions of urinary mucosa (white arrow). (b) Microscopic view of the ulcerated surface of the polypoid lesion (H-E stain X 2.5).

between $10 \%$ and $30 \%$ at a mean follow-up time of approximately 30 months $[6,7]$. Although radical cystectomy is an aggressive therapeutic option, minimizing the risk of grade and stage progression may optimize patient's longterm outcome.

\section{Consent}

Written informed consent was obtained from the patient for publication of this paper and accompanying images. A copy of the written consent is available for review by the Editorin-Chief of this journal.

\section{Abbreviations}

IMT: Inflammatory myofibroblastic tumor

IP: Inflammatory pseudotumor

PSCN: Postoperative spindle cell nodule
CT: $\quad$ Computed tomography

$\alpha$-SMA: $\alpha$-Smooth muscle actine

NSE: Neuron-specific enolase

SFT: $\quad$ Solitary fibrous tumors

ALK: Anaplastic lymphoma kinase

NF-1: Neurofibromatosis type-1.

\section{References}

[1] L. Cheng, S. R. Foster, G. T. MacLennan, A. Lopez-Beltran, S. Zhang, and R. Montironi, "Inflammatory myofibroblastic tumors of the genitourinary tract-single entity or continuum?” Journal of Urology, vol. 180, no. 4, pp. 1235-1240, 2008.

[2] J. A. Roth, "Reactive pseudosarcomatous response in urinary bladder," Urology, vol. 16, no. 6, pp. 635-637, 1980.

[3] K. H. Proppe, R. E. Scully, and J. Rosai, "Postoperative spindle cell nodules of genitourinary tract resembling sarcomas. A report of eight cases," American Journal of Surgical Pathology, vol. 8, no. 2, pp. 101-108, 1984. 
[4] A. Lopez-Beltran, J. Lopez-Ruiz, and L. Vicioso, "Inflammatory pseudotumor of the urinary bladder-a clinicopathological analysis of two cases," Urologia Internationalis, vol. 55, no. 3, pp. 173-176, 1995.

[5] H. Hojo, W. A. Newton Jr., A. B. Hamoudi et al., "Pseudosarcomatous myofibroblastic tumor of the urinary bladder in children: a study of 11 cases with review of the literature. An Intergroup Rhabdomyosarcoma Study," American Journal of Surgical Pathology, vol. 19, no. 11, pp. 1224-1236, 1995.

[6] E. A. Montgomery, D. D. Shuster, A. L. Burkart et al., "Inflammatory myofibroblastic tumors of the urinary tract: a clinicopathologic study of 46 cases, including a malignant example inflammatory fibrosarcoma and a subset associated with high-grade urothelial carcinoma," American Journal of Surgical Pathology, vol. 30, no. 12, pp. 1502-1512, 2006.

[7] L. R. Harik, C. Merino, J.-M. Coindre, M. B. Amin, F. Pedeutour, and S. W. Weiss, "Pseudosarcomatous myofibroblastic proliferations of the bladder: a clinicopathologic study of 42 cases," American Journal of Surgical Pathology, vol. 30, no. 7, pp. 787-794, 2006.

[8] M. D. Blum, R. R. Bahnson, and M. F. Carter, "Urologic manifestations of von Recklinghausen neurofibromatosis," Urology, vol. 26, no. 3, pp. 209-217, 1985.

[9] S. Poleksic, "Leiomyosarcoma of urinary bladder in von Recklinghausen's neurofibromatosis," Urology, vol. 10, no. 4, pp. 341-342, 1977.

[10] T. L. Däuth, M. Conradie, and R. Chetty, "Leiomyoma of the bladder in a patient with von Recklinghausen's neurofibromatosis," Journal of Clinical Pathology, vol. 56, no. 9, pp. 711712, 2003. 


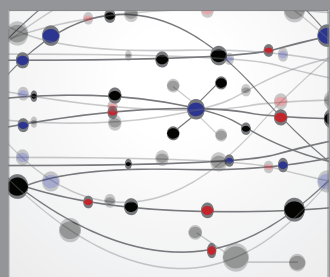

The Scientific World Journal
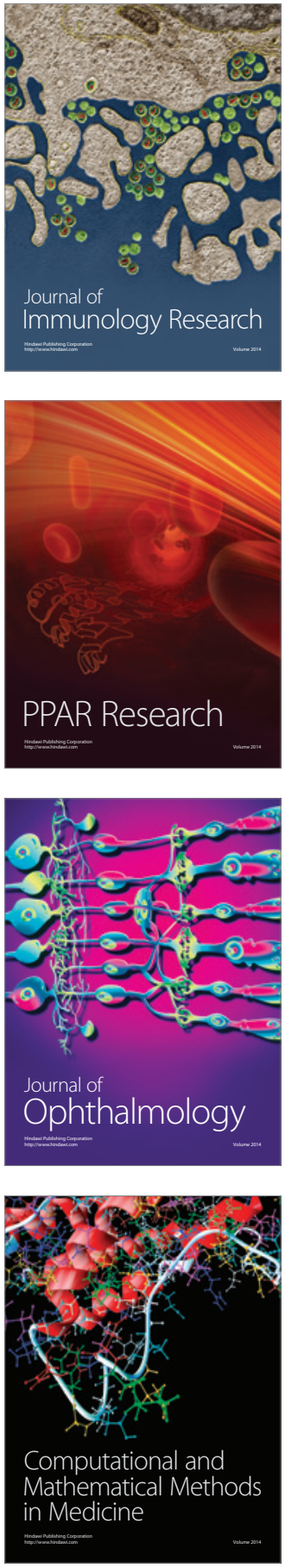

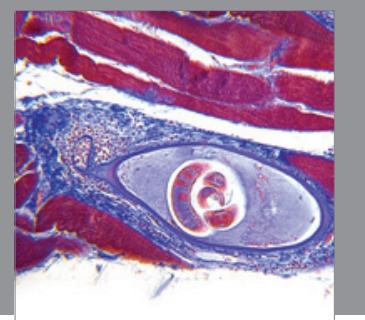

Gastroenterology

Research and Practice
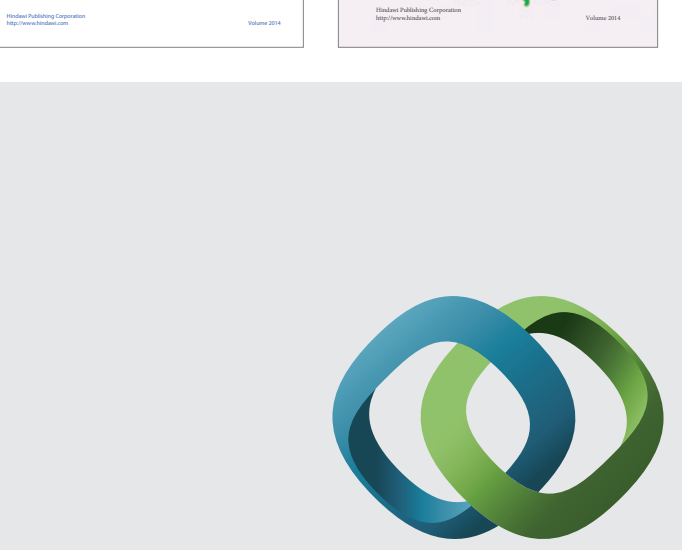

\section{Hindawi}

Submit your manuscripts at

http://www.hindawi.com
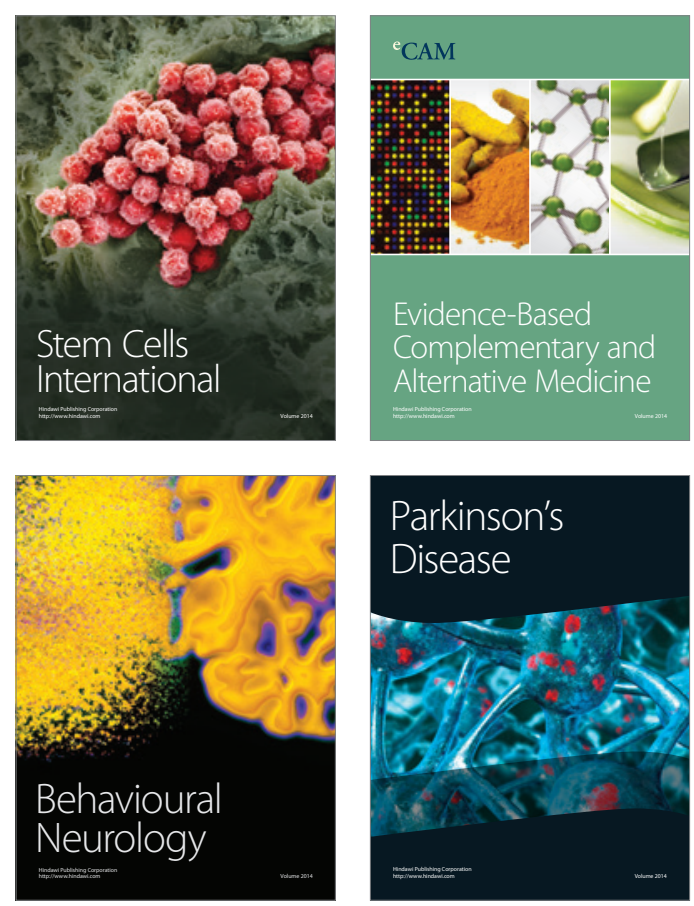

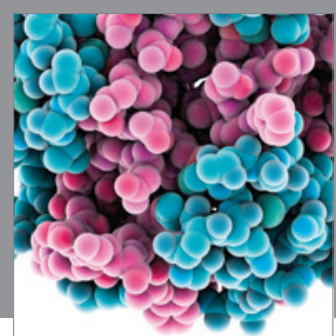

Journal of
Diabetes Research

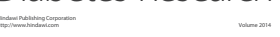

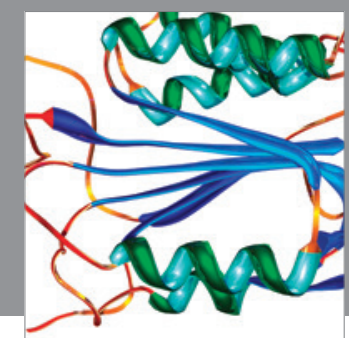

Disease Markers
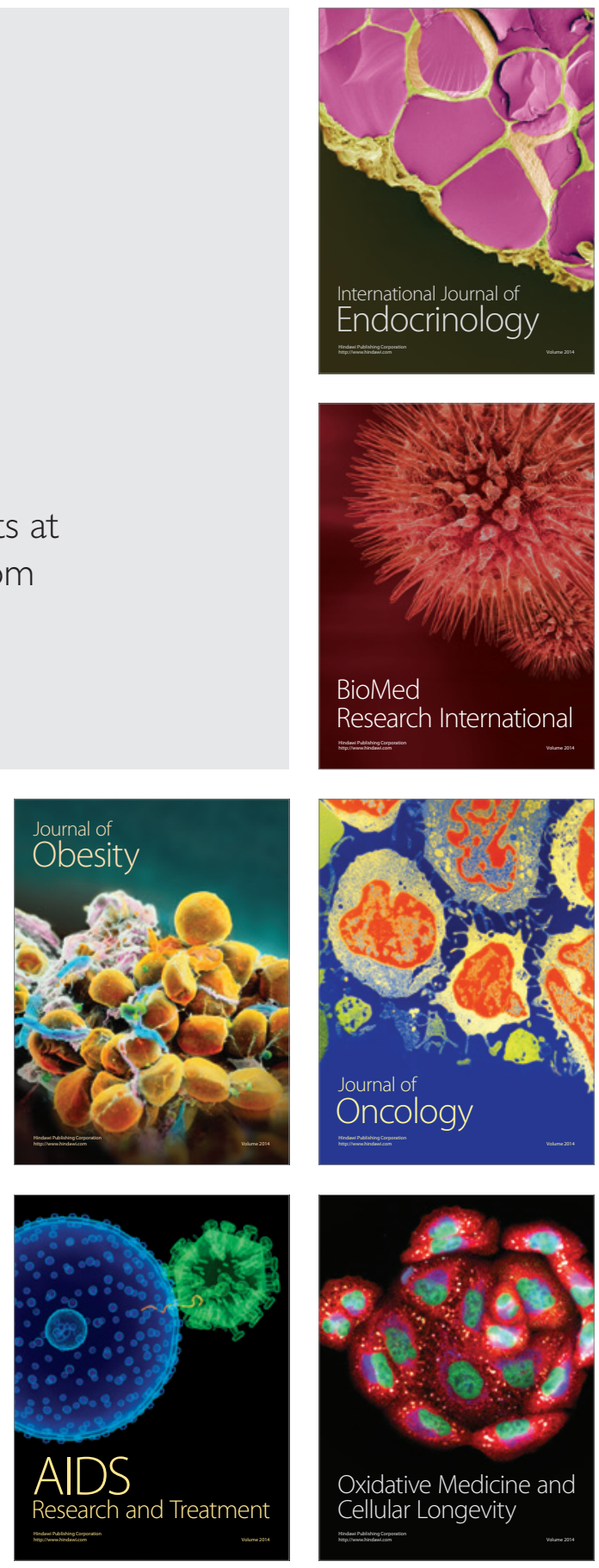